\title{
Different methods to calculate genomic predictions-Comparisons of BLUP at the single nucleotide polymorphism level (SNP-BLUP), BLUP at the individual level (G-BLUP), and the one-step approach (H-BLUP)
}

\author{
M. Koivula, ${ }^{, 1}$ I. Strandén, ${ }^{*}$ G. Su,, and E. A. Mäntysaari ${ }^{*}$ \\ *MTT Agrifood Research Finland, Biotechnology and Food Research, Biometrical Genetics Fl-31600 Jokioinen, Finland \\ †Department of Genetics and Biotechnology, Faculty of Science and Technology, Aarhus University, DK-8830 Tjele, Denmark
}

\begin{abstract}
Several strategies to use genomic data in predictions have been proposed. The aim of this study was to compare different genomic prediction methods. The response variables used in the genomic predictions were deregressed proofs, which were derived from 2 estimated breeding value (EBV) data sets. The full EBV data set from March 2010 included the EBV for production and mastitis traits for all Nordic red bulls. The reduced data set included the same animals as the full data set, but the EBV were predicted from a data set that excluded the last 5 yr of observations. Genomic predictions were obtained using different BLUP models: BLUP at the single nucleotide polymorphism level (SNP-BLUP), BLUP at the individual level (GBLUP), and the one-step approach (H-BLUP). For the selection candidate bulls, the SNP-BLUP and G-BLUP models gave the same direct genomic breeding values (e.g., correlation of direct genomic breeding values between SNP-BLUP and G-BLUP for protein was 0.99), but slightly different from genomic EBV obtained from H-BLUP (correlations of SNP-BLUP or G-BLUP with H-BLUP were about 0.96). For all traits, SNPBLUP and G-BLUP gave the same validation reliability, whereas H-BLUP led to slightly higher reliability. Therefore, the results support a slight advantage of using H-BLUP for genomic evaluation.
\end{abstract}

Key words: genetic evaluation, genomic selection, genomic breeding value, BLUP

\section{INTRODUCTION}

Genomic selection refers to genetic improvement of animals or plants through selection based on evaluations utilizing genomic information. Genomic evaluations can be either direct genomic values (DGV) pre-

Received August 26, 2011.

Accepted February 18, 2012.

${ }^{1}$ Corresponding author: minna.koivula@mtt.fi dicted using a reference population of animals that have genotype as well as phenotypic information (Meuwissen et al., 2001), or genomically enhanced breeding values (GEBV). The GEBV are obtained by combining DGV with the extra information included in traditional EBV calculation but not in phenotypes of reference animals (VanRaden et al., 2009). Genomic selection is currently widely applied in dairy cattle breeding (Hayes et al., 2009a; VanRaden et al., 2009; Harris and Johnson, 2010; Su et al., 2010; VanRaden and Sullivan, 2010). The increasing availability of marker information in livestock combined with improvements in genomic selection methods allow breeders to select animals on genotypes early in life. The use of pedigree, phenotypic, and genotypic data in combination can increase genetic progress by decreasing the generational interval and by increasing the accuracy of genomic predictions and thus reliability of assessment of animals' genetic merit. Because genotyping is costly and not all animals are genotyped, most strategies for genomic selection are currently based on a multi-step procedure (Guillaume et al., 2008; VanRaden, 2008; Hayes et al., 2009a; VanRaden et al., 2009). In the first step, breeding values are estimated by traditional methods without genotypic information. In the second step, corrected phenotypes or pseudo-data with genomic information are used to calculate estimates of DGV. If required, in a third step DGV are combined with EBV, although discounting for (male) parent average (PA) information already in DGV.

Many statistical models have been proposed to predict DGV (e.g., Meuwissen et al., 2001; Meuwissen and Goddard, 2004; Gianola et al., 2006). Genomic predictions can be performed by estimating marker effects or by using mixed model equations with genomic relationship matrix G (Meuwissen et al., 2001; VanRaden, 2008; Goddard, 2009; Strandén and Garrick, 2009). In random regression SNP marker models (later called SNP-BLUP), the marker effects are estimated with BLUP, assuming a normal distribution and equal variance for all markers. Subsequently, DGV can be 
calculated by summing the effects of the alleles across all markers. Goddard (2009) and Strandén and Garrick (2009) showed that SNP-BLUP is equivalent to a model in which the breeding values are estimated from the data directly as with traditional BLUP, but by using a genomic relationship matrix rather than a pedigreebased relationship matrix. Because the genomic relationship matrix is usually named $\mathbf{G}$, hereafter a BLUP using a $\mathbf{G}$ matrix will be called G-BLUP.

Linear BLUP models (at either the SNP level or the individual animal level) have become popular approaches in practical genomic evaluations because they are simple and have low computational requirements (Meuwissen et al., 2001; VanRaden, 2008; Goddard and Hayes, 2009; Hayes et al., 2009b). Results in dairy cattle have indicated that using a large number of markers with equal variance for all markers is appropriate for most traits. Only a slight decrease in accuracy has occurred for some traits by assuming equal rather than different variance for each SNP marker (Cole et al., 2009; VanRaden et al., 2009). Villanueva et al. (2005) also reported that genomic relationships constructed using a large number of markers increase reliability of genomic prediction. In addition, assuming equal variance allows the use of the same genomic relationship matrix for all traits. Genomic relationship matrix $\mathbf{G}$ can be constructed in several ways (Gianola and van Kamm, 2008; VanRaden, 2008), and various G matrices used in a genetic evaluation have resulted in different scaling and accuracies of GEBV (Aguilar et al., 2010; Forni et al., 2011).

The accuracies of genomic predictions can be improved by combining genomic information and information from traditional EBV. VanRaden et al. (2009) proposed to generate GEBV by a selection index that includes DGV, traditional EBV, PA, or pedigree indexes (PI, the both-parent average) calculated from the whole population, and PA (or PI) calculated from the data of genotyped animals. A one-step genetic evaluation based on the integration of the pedigree relationship matrix and genomic information into a single H matrix has been proposed by Legarra et al. (2009), Misztal et al. (2009), and Christensen and Lund (2010). Aguilar et al. (2010) and Christensen and Lund (2010) independently described the inverse of such a joint relationship matrix. This type of one-step approach has high computational demand in the cases of large data set and multi-trait analysis and when the reliabilities of individual GEBV are required ( $\mathrm{Su}$ et al., 2012). However, the use of deregressed proofs (DRP) of bulls as the response variable, instead of using uncorrected raw data, is a compromise between high computational demand and full use of all data. The generated genomic predictions are equivalent to GEBV but are obtained directly. Garrick et al. (2009) and Ostersen et al. (2011) have shown that DRP with PA removed may yield more accurate genomic predictions. Another advantage of using DRP in the one-step approach could be in cases where the raw data are not available to compute genomic EBV (e.g., regional or multiple-country evaluations).

The objectives of this study were to apply a simple marker model (SNP-BLUP), alternative definitions of genomic matrices in G-BLUP, and one-step BLUP $(\mathbf{H}-$ BLUP) to analyses of production and mastitis traits in Nordic Red Dairy cattle (RDC). We wanted to compare the predictions and reliabilities of direct genetic values from SNP-BLUP, G-BLUP, and H-BLUP and to test if the one-step approach could improve genetic evaluation for nongenotyped young candidate bulls.

\section{MATERIALS AND METHODS}

\section{Marker Data and G Matrix}

The RDC bulls were genotyped using the Illumina Bovine SNP50 BeadChip (Illumina, San Diego, CA). Markers on the X chromosome and without a map position in the Btau 4.0 assembly were discarded. Individual genotypes with a GenCall score (Illumina Inc., 2005) of $<0.6$ were discarded. Missing genotypes were imputed using the fastPHASE software (Scheet and Stephens, 2006). After the editing, marker data included 6,145 genotyped bulls with 37,996 SNP markers.

\section{Phenotypic Data}

Two data sets, full and reduced, were obtained from official Nordic genetic evaluations for the RDC. The full data set from March 2010 included the EBV, their reliabilities $\left(r_{E B V}^{2}\right)$, and effective daughter contributions (EDC; Fikse and Banos, 2001) for all bulls. The reduced data set had the same animals as the full data, but EBV were calculated using data until 2005 (i.e., 5 yr of observations were deleted). The reduced data were used as reference data to predict genomic breeding values. Bulls that had EBV based on at least 10 daughters with records in the full data but had only PA information in the reduced data set were defined as candidate bulls.

The EBV of milk, protein, and fat were used to represent production trait evaluations, and mastitis was chosen as an example of low heritable health trait. A summary of data is shown in Table 1 . The response variables used in the genomic predictions were DRP (Goddard 1985; Jairath et al., 1998; Schaeffer, 2001; Garrick et al., 2009). The DRP for all traits were ob- 
Table 1. Number of bulls in different data sets by trait

\begin{tabular}{lcccccc}
\hline & \multicolumn{2}{c}{ Full data } & & \multicolumn{2}{c}{ Reference bulls } & \\
\cline { 2 - 3 } \cline { 5 - 6 } Trait & $\begin{array}{c}\text { Bulls with } \\
\text { DRP }\end{array}$ & $\begin{array}{c}\text { Genotyped } \\
\text { with DRP }\end{array}$ & & $\begin{array}{c}\text { Bulls with } \\
\text { DRP }\end{array}$ & $\begin{array}{c}\text { Genotyped } \\
\text { with DRP }\end{array}$ & $\begin{array}{c}\text { Candidate } \\
\text { bulls }\end{array}$ \\
\hline Milk & 6,253 & 4,145 & & 5,313 & 3,330 & 809 \\
Protein & 6,253 & 4,145 & & 5,313 & 3,330 & 809 \\
Fat & 6,253 & 4,145 & & 5,313 & 3,330 & 809 \\
Mastitis & 6,169 & 4,431 & & 5,363 & 3,649 & 780 \\
\hline 1. & & & & &
\end{tabular}

${ }^{1}$ Deregressed proofs.

tained by applying an iterative procedure, as described by Jairath et al. (1998) and Schaeffer (2001), and currently used by Interbull (Uppsala, Sweden). The calculations were done using option DeRegress (Strandén and Mäntysaari, 2010) in MiX99 package (Strandén and Lidauer, 1999). Heritabilities for the deregression procedure were those reported to Interbull for the Nordic RDC (Table 2). The differing number of daughters per sire contributing to the calculation of DRP was accounted for by using EDC as a weighting factor. The EBV of all bulls in the pedigree were included in the deregression procedure. The DRP was accepted as an observation in the model if its reliability $\left(r_{D R P}^{2}\right)$ was $>20 \%$. The $r_{D R P}^{2}$ for bull $i$ was estimated as $r_{D R P, i}^{2}=E D C_{i} /\left(E D C_{i}+\lambda\right)$, where $\lambda=\left(4-h^{2}\right) / h^{2}$. The DRP were scaled so that mean of DRP in data sets was zero.

\section{Statistical Analyses}

Breeding values were estimated using the following BLUP models, based on the reduced data. The SNP marker information was the same in all statistical analyses. The SNP marker information can be arranged to an $n$ by $m$ model design matrix $\mathbf{M}$, where $n$ is number of animals and $m$ is number of markers. Thus, a row has genotypes that an individual has inherited. A column has SNP markers for all individuals coded 0,1 , and 2 for homozygote (AA), heterozygote (Aa), and the other homozygote (aa), respectively (alleles arbitrarily called A and a).

The first model was SNP-BLUP:

$$
\mathbf{y}=1 \mu+\mathbf{M g}+\mathbf{e}
$$

where $\mathbf{y}$ is the vector of DRP, $\mu$ is the intercept, $\mathbf{g}$ is the vector of random unknown marker effects, and $\mathbf{e}$ is the vector of random residuals. The marker effects $\mathbf{g}_{i}$ were assumed to be identically and independently distributed with mean zero and variance $\sigma_{g}^{2}$; that is, $\mathbf{g} \sim N\left(0, \mathbf{I} \sigma_{g}^{2}\right)$.
All BLUP models assumed that $\mathbf{e} \sim N\left(0, \mathbf{D} \sigma_{e}^{2}\right)$. The diagonal element in matrix $\mathbf{D}$ was $d_{i i}=1 / w_{i}$, where $w_{i}$ $=\mathrm{EDC}_{\mathrm{i}} / \lambda$ is a weighting factor for the $i$ th DRP. The weighting factor accounted for heterogeneous residual variances due to the difference in reliabilities of DRP (VanRaden, 2008; Garrick et al., 2009). Estimated solutions from the SNP-BLUP were used to estimate DGV by $\hat{\mathbf{a}}=\mathbf{1} \hat{\mu}+\mathbf{M} \hat{\mathbf{g}}$.

The model used in G-BLUP was equivalent to SNPBLUP but instead of estimating the SNP effects only the sum of the SNP effects $\mathbf{a}=\mathbf{Z g}$ corresponding the DGV above were estimated. Let matrix $\mathbf{P}$ contain allele frequencies of the second allele, $p_{i}$, calculated from the marker data of all genotyped bulls. Then, let $\mathbf{Z}=\mathbf{M}$ - 2P. An unscaled genomic relationship matrix is $\mathbf{G}_{0}$ $=\mathbf{Z Z}$ '. Following VanRaden (2008), the scaled genomic matrix is $\mathbf{G}_{\mathrm{k}}=\mathbf{Z} \mathbf{Z}^{\prime} / k$, with a scaling parameter $k=$ $2 \Sigma p_{i}\left(1-p_{i}\right)$. The scaling makes $\mathbf{G}_{\mathrm{k}}$ similar to traditional numerator relationship matrix $\mathbf{A}$. The G-BLUP had either a plain genomic relationship matrix $\left(\mathbf{G}_{0^{-}}\right.$ BLUP) or a scaled matrix $\left(\mathbf{G}_{\mathrm{k}}-\mathbf{B L U P}\right)$. The basic G-BLUP model was

$$
\mathbf{y}=\mathbf{1} \mu+\mathbf{X a}+\mathbf{e},
$$

where $\mathbf{a}$ is the vector of individual $\mathrm{DGV}$, and $\mathbf{X}$ is an incidence matrix relating DGV to corresponding observation. Genetic effects were assumed to follow a normal distribution $\mathbf{a} \sim N\left(0, \mathbf{G} \sigma_{a k}^{2}\right)$, where $\sigma_{a k}^{2}$ is genetic vari-

Table 2. Heritability $\left(\mathrm{h}^{2}\right), \lambda\left[\lambda=\left(4-\mathrm{h}^{2}\right) / \mathrm{h}^{2}\right]$, and mean reliability of deregressed proofs $\left(r^{2}\right.$ DRP $)$ for reference and candidate bulls by trai

\begin{tabular}{lcccc}
\hline & & \multicolumn{2}{c}{$\mathrm{r}^{2}{ }_{\mathrm{DRP}}$} \\
\cline { 3 - 5 } Trait & $\mathrm{h}^{2}$ & \multicolumn{1}{c}{$\lambda$} & $\begin{array}{c}\text { Reference } \\
\text { bulls }\end{array}$ & $\begin{array}{c}\text { Candidate } \\
\text { bulls }\end{array}$ \\
\hline Milk & 0.39 & 9.26 & 0.95 & 0.93 \\
Protein & 0.31 & 11.90 & 0.96 & 0.94 \\
Fat & 0.36 & 10.11 & 0.96 & 0.93 \\
Mastitis & 0.04 & 99.00 & 0.88 & 0.80 \\
\hline
\end{tabular}


ance, and $\mathbf{G}$ is the genomic relationship matrix $\left(\mathbf{G}_{0}\right.$ or $\mathbf{G}_{\mathrm{k}}$ ).

The one-step approach (H-BLUP) following Legarra et al. (2009), Aguilar et al. (2010), and Christensen and Lund (2010), was based on the model

$$
\mathbf{y}_{\mathbf{t}}=1 \mu+\mathbf{W a}+\mathbf{e},
$$

where $\mathbf{y}_{\mathbf{t}}$ is a vector of DRP of all bulls, including nongenotyped bulls, $\mathbf{a}$ is the vector of additive genetic effects, and $\mathbf{W}$ is incidence matrix relating the breeding values a to corresponding observations $\mathbf{y}_{\mathrm{t}}$. Genetic values were assumed to follow a normal distribution, $\mathbf{a} \sim N\left(0, \mathbf{H} \sigma_{a}^{2}\right)$, where $\sigma_{a}^{2}$ is additive genetic variance and $\mathbf{H}$ is the relationship matrix combining both SNP marker and pedigree information.

In $\mathbf{H}$, the variance-covariance structure of genotyped animals was taken from the genomic relationship matrix and the relationships of nongenotyped animals were "corrected" with respect to differences in genomic and pedigree-based relationships of their genotyped relatives. The inverse of the $\mathbf{H}$ matrix has a simple structure (Aguilar et al., 2010; Christensen and Lund, 2010):

$$
\mathbf{H}^{-1}=\mathbf{A}^{-1}+\left[\begin{array}{cc}
\mathbf{G}_{\boldsymbol{w}}^{-1}-\mathbf{A}_{11}^{-1} & 0 \\
0 & 0
\end{array}\right],
$$

where $\mathbf{A}_{11}$ is the sub-matrix of $\mathbf{A}$ (numerator relationship matrix) for the genotyped animals, and $\mathbf{G}_{\mathbf{w}}=w t$ $\mathbf{G}_{\mathbf{k}}+(1-w) \mathbf{A}_{11}$ is the relationship matrix constructed using genomic and pedigree information. The constant $w$ is proportion of additive genetic variance accounted by the SNP markers from the total additive genetic variance. We used $w=0.90$, which assumes that $10 \%$ of total genetic variance is due to the polygenic effect not described by the SNP markers. The correction factor $t$ was used to scale $\mathbf{G}_{\mathbf{k}}$ so that the diagonals in $\left(\mathrm{t} \mathbf{G}_{\mathbf{k}}\right)$ and A were, on average, equal before the matrices $\left(\mathrm{t} \mathbf{G}_{\mathrm{k}}\right)$ and $\mathbf{A}_{11}$ were combined.

Variance of marker effects $\left(\sigma_{g}^{2}\right)$ and residual variance $\left(\sigma_{e}^{2}\right)$ were estimated from the full data using a SNP marker genetic model (model [1]) with a Bayesian method. These estimated variance components were used in SNP-BLUP and $\mathrm{G}_{0}$-BLUP to predict genomic breeding values. For $\mathrm{G}_{\mathrm{k}}$-BLUP and H-BLUP, the genetic variance $\left(\sigma_{a}^{2}\right)$ was obtained by multiplying the estimated marker genetic variance by the scaling factor $k$. Because H-BLUP uses both the pedigree and the genomic information, a plausible alternative is to use the variance components used in national evaluation (Table
2 ). For comparison, H-BLUP was done with both variance components. All the BLUP analyses were carried out using MiX99 (Strandén and Lidauer, 1999), which uses preconditioned conjugate gradient iteration. The H-BLUP implementation in MiX99 constructs the $\mathbf{A}^{-1}$ matrix directly by reading the pedigree file while iterating on data and reads the $\mathbf{G}_{w}^{-1}-\mathbf{A}_{11}^{-1}$ matrix block of the $\mathbf{H}$ matrix from a separate file during each preconditioned conjugate gradient iteration cycle.

Genomic variance ratio was derived from the variance component estimates of marker genetic model. These were defined $\left[\hat{h}_{g}^{2}=4 \hat{\sigma}_{a}^{2} /\left(\hat{\sigma}_{a}^{2}+\lambda \hat{\sigma}_{e}^{2}\right)\right]$, where $\hat{\sigma}_{a}^{2}$ is an estimate of additive genetic variance and $\hat{\sigma}_{e}^{2}$ is an estimate of residual variance. The residual variance estimate was back scaled by multiplying with $\lambda$ because the scaled weights were used in model [1]. Genetic variance, $\sigma_{a}^{2}$, was $k \sigma_{g}^{2}$, where $k$ is the scaling parameter, and $\sigma_{g}^{2}$ is an estimate of SNP marker variance from Bayesian SNP analysis.

\section{Validation of Genomic Prediction}

Validation reliability of predictions (DGV and GEBV) was assessed using Interbull validation protocol (Mäntysaari et al., 2010):

$$
\mathbf{y}=\mathbf{1} b_{0}+b_{1} \hat{a}+\mathbf{e}
$$

where $\mathbf{y}$ are the DRP of the candidate bulls in the full data, and $\hat{\mathbf{a}}$ are the genomic prediction for these bulls from the analysis based on the reduced data. The reliabilities of DRP $\left[\mathrm{r}^{2}{ }_{\mathrm{DRPi}}=\mathrm{EDC}_{\mathrm{i}} /\left(\mathrm{EDC}_{\mathrm{i}}+\lambda\right)\right]$ were used as weights. The validation reliability of the model was obtained from the $\mathrm{R}^{2}$ (coefficient of determination) of the model [4], after correcting it by the average reliability of DRP of the candidate bulls; that is, $R_{\text {validation }}^{2}=R_{\text {model }}^{2} / \bar{r}_{D R P}^{2}$. Unbiasedness of genomic predictions was assessed by the coefficients of the regression model $\left(b_{0}\right.$ and $\left.b_{1}\right)$. A necessary (but not sufficient) condition for unbiased prediction is that the regression intercept $b_{0}$ does not deviate significantly from the mean of EBV. Moreover, this can be true only if the regression coefficient $b_{1}$ does not deviate from 1 . To estimate the further gain from the genomic information over the traditional PA (VanRaden et al., 2009; Mäntysaari et al., 2010), the same validation test was also applied to PA EBV.

To test the gain in prediction accuracy of the nongenotyped candidates in H-BLUP, a sample of candidates was re-evaluated without their own genomic information. The marker information of the 200 youngest candidate bulls was removed, and $\mathbf{H}^{-1}$ was constructed 
using the remaining genotypes. The 200 nongenotyped candidate bulls still had the pedigree information. The 200 bulls had indirectly genomic information through their sires, as 32 out of the 35 sires had been genotyped.

\section{RESULTS AND DISCUSSION}

The number of bulls in the data sets is given in Table 1. The data set included 809 candidate bulls for production traits and 780 for mastitis. The number of reference bulls in SNP-BLUP and G-BLUP was 3,330 for production traits and 3,649 for mastitis. In H-BLUP, all bulls with DRP in the reduced data were used as reference bulls.

Heritability and lambda [where $\lambda=\left(4-\mathrm{h}^{2}\right) / \mathrm{h}^{2}$ ] values used in the deregression are in Table 2. These are the population parameters reported to Interbull for Nordic RDC. The average reliabilities of DRP were higher for the reference bulls than for the candidate bulls for most traits. This is because candidate bulls were younger and had fewer daughters with records or fewer daughters with later lactation records. Average reliability of DRP was $94 \%$ in the reference bulls and $90 \%$ in the candidate bulls. The genomic variance ratios estimated by Bayesian variance component analysis were $36.0,37.5,35.0$, and 362.6 for milk, protein, fat, and mastitis, respectively. These values were considerably higher than the original lambda values presented in Table 2. This could be because of different definitions of genetic and genomic variance, but also because the sample of genotyped bulls is a selected sample of all bulls. The reason for the high variance ratio, however, seems to be overestimation of the residual variance in the genomic model.

The correlations between genomic predictions from different methods are presented in Table 3. For the reference bulls, the correlations between SNP-BLUP, $\mathrm{G}_{\mathrm{k}}$-BLUP, and $\mathrm{G}_{0}$-BLUP were close to 1 for all traits. For the candidate bulls, the correlations were slightly lower than for reference bulls, but close to 1 . Thus, the results confirmed that different methods performed very similarly, and DGV did not differ greatly whether using a plain or a scaled genomic relationship matrix. The small departures of correlations from unity are most likely due to different convergence characteristics of the models, because all solutions were calculated by an iterative solving method. The close unity correlations support derivations by Strandén and Christensen (2011), which proved that when the fixed general mean is in the model, there are no differences in inference by the alternative genomic relationship matrices tested. In the current study, only predictions from H-BLUP differed slightly from the others and the correlations were lower, both in reference and candidate bulls. This was expected because the H-BLUP uses pedigree information and observations of both genotyped and nongenotyped animals to estimate genomic predictions, whereas other methods use only marker information and observations of genotyped animals. The assumption that $10 \%$ of total genetic variance was due to the polygenic effect is one reason to differences in genomic predictions of H-BLUP from others. In addition, HBLUP generates GEBV, not DGV, which explains the differences. The difference between the two would be even greater if the GEBV directly used the information from female relatives. In our study, the phenotypic records were bull DRP, thus the bull dam information was included only via bull EBV. Correlations between PA and different genomic models are clearly lower than correlations among genomic models. However, correlations between H-BLUP and PA were higher than correlations between PA and SNP-BLUP or G-BLUP. This can be expected because H-BLUP estimates contain PA directly, whereas DGV generated by SNP-BLUP or G-BLUP contains PA only indirectly through genomic relationships of candidates and reference population but ignoring phenotypic information from non-genotyped ancestors.

The SNP-BLUP and the 2 G-BLUP methods seemed to give the same results. Thus, the choice of implementation can be based on practical issues. The advantage of SNP-BLUP is that it offers an easy path to automate the DGV calculation of new candidates as soon as their genotypes are obtained from the laboratory. The DGV from G-BLUP could also be transformed into SNP solution using equations from Strandén and Garrick (2009) and Yang et al. (2011). The advantage of G-BLUP methods is that they offer a computationally easier way to calculate prediction error variances, as long as the number of animals is smaller than the number of SNP markers. A disadvantage of G-BLUP methods is the need of dense inverse of the $\mathbf{G}$ matrix and possibly the inverse of the MME coefficient matrix. Computing time did not differ much with the different BLUP models. The most time-consuming part in G-BLUP and H-BLUP are the construction and inverse of the $\mathbf{G}$ matrix and the $\mathbf{H}$ matrix. In MiX99, the $\mathrm{G}_{\mathrm{k}}$-BLUP model needed fewer iterations and less time than the other BLUP models. However, in practice, the computing time differences between the models were negligible, as the $\mathrm{G}_{\mathrm{k}}$-BLUP converged in about $50 \mathrm{~s}$ and SNPBLUP in $5 \mathrm{~min}, \mathrm{G}_{0}$-BLUP in $9 \mathrm{~min}$, and H-BLUP in 3 min (with a 2.8-GHz Intel Xeon processor). Building and inverting the $\mathbf{G}$ matrix took about 20 min. Inversions of matrices were computed using the generalized inverse algorithm from the BLUPf90 package (Misztal et al., 2002), which uses the subroutines from LAPACK 
Table 3. Correlations between genomic predictions from SNP-BLUP, $\mathrm{G}_{\mathrm{k}}$-BLUP, $\mathrm{G}_{0}$-BLUP, H-BLUP, and parent-average BLUP (PA) for the reference and the candidate bulls by trait

\begin{tabular}{|c|c|c|c|c|c|c|c|c|c|}
\hline \multirow[b]{2}{*}{ Trait } & \multirow[b]{2}{*}{ Model $^{1}$} & \multicolumn{4}{|c|}{ Reference bulls } & \multicolumn{4}{|c|}{ Candidate bulls } \\
\hline & & $\mathrm{G}_{\mathrm{k}}$-BLUP & $\mathrm{G}_{0}$-BLUP & H-BLUP & $\mathrm{PA}$ & $\mathrm{G}_{\mathrm{k}}-\mathrm{GBLUP}$ & $\mathrm{G}_{0}$-BLUP & H-BLUP & PA \\
\hline \multirow{3}{*}{ Milk } & $\mathrm{G}_{\mathrm{k}}-\mathrm{BLUP}$ & & 0.999 & 0.998 & 0.963 & & 0.999 & 0.971 & 0.680 \\
\hline & $\mathrm{G}_{0}$-BLUP & & & 0.998 & 0.961 & & & 0.971 & 0.678 \\
\hline & H-BLUP & & & & 0.965 & & & & 0.763 \\
\hline \multirow{2}{*}{ Protein } & SNP-BLUP & 0.999 & 0.999 & 0.997 & 0.961 & 0.999 & 0.999 & 0.962 & 0.653 \\
\hline & H-BLUP & & & & 0.967 & & & & 0.752 \\
\hline \multirow[t]{4}{*}{ Fat } & SNP-BLUP & 0.999 & 0.999 & 0.998 & 0.969 & 0.999 & 0.999 & 0.978 & 0.656 \\
\hline & $\mathrm{G}_{\mathrm{k}}-\mathrm{BLUP}$ & & 0.999 & 0.998 & 0.971 & & 0.999 & 0.979 & 0.658 \\
\hline & $\mathrm{G}_{0}-\mathrm{BLUP}$ & & & 0.998 & 0.969 & & & 0.979 & 0.656 \\
\hline & H-BLUP & & & & 0.975 & & & & 0.730 \\
\hline \multirow{2}{*}{ Mastitis } & SNP-BLUP & 0.999 & 0.999 & 0.979 & 0.892 & 0.999 & 0.999 & 0.984 & 0.663 \\
\hline & $\mathrm{G}_{\mathrm{k}}-\mathrm{BLUP}$ & & 0.999 & 0.979 & 0.892 & & 0.999 & 0.984 & 0.660 \\
\hline
\end{tabular}

${ }^{1} \mathrm{SNP}-\mathrm{BLUP}=\mathrm{SNP}$ marker BLUP model; $\mathrm{G}_{\mathrm{k}}$-BLUP $=$ BLUP using a scaled genomic relationship matrix; $\mathrm{G}_{0}$-BLUP $=$ BLUP using an unscaled genomic relationship matrix; and H-BLUP = one-step approach.

(Anderson et al., 1990). However, for large matrices, as here, use of optimized LAPACK routines can reduce the computing time considerably (Aguilar et al., 2011).

Table 4 presents the regression coefficients and validation reliabilities for the candidate bulls from the Interbull validation protocol. As suggested by the correlations among tested methods, the differences in validation results for SNP-BLUP and both G-BLUP methods were within rounding errors. As the general mean was added to genomic predictions, all the methods gave roughly same validation intercepts. For PA, the $\mathrm{b}_{0}$ terms were significantly greater than zero $(2.34$ to 4.26) for production traits but close to zero for mastitis. This shows that the mean breeding value for young sires is not exactly the mean breeding value of their male ancestors but is slightly higher. Bull dams are a selected group from all daughters of maternal grandsires and the selection seems to be more on production than on health.
The regression coefficients $\left(b_{1}\right)$ for SNP-BLUP and G-BLUP-methods were between 0.76 and 0.78 for milk, protein, and mastitis but higher $(\sim 0.85)$ for fat (Table 4 ). This suggests that DGV overpredict the variation in DRP. The overdispersion seems to be very similar with PA, except for mastitis, where PA showed greater overdispersion than SNP-BLUP and G-BLUP. The $b_{1}$ from H-BLUP were generally higher especially when using estimated covariance components. This suggests that GEBV by H-BLUP are less biased than DGV.

The validation reliabilities for SNP-BLUP and GBLUP methods were 0.30 to 0.31 for milk and protein but higher (0.40) for fat. Higher reliability of fat prediction has been reported elsewhere ( $\mathrm{Su}$ et al., 2012). Most likely, this is caused by a known large effect of the $D G A T$ gene with a large effect on fat percentage (Grisart et al., 2004). The validation reliability was lower for mastitis (0.17) than for the production traits. The increase in $\mathrm{R}_{\text {validation }}^{2}$ with DGV over the PA was 11 per-

Table 4. Intercepts $\left(b_{0}\right)$, regression coefficients $\left(b_{1}\right)$, and validation reliabilities $\left(R^{2}\right)$ of direct genomic breeding values from SNP-BLUP, $G_{k}-$ BLUP, or $\mathrm{G}_{0}$-BLUP, and genomic breeding values from H-BLUP with estimated variance components $\left(\mathrm{H}_{-} \mathrm{BLUP}_{\mathrm{e}}\right.$ ) and variance components from the national model $\left(\mathrm{H}-\mathrm{BLUP}_{\mathrm{I}}\right)$, and conventional parent averages $(\mathrm{PA})$ by trait

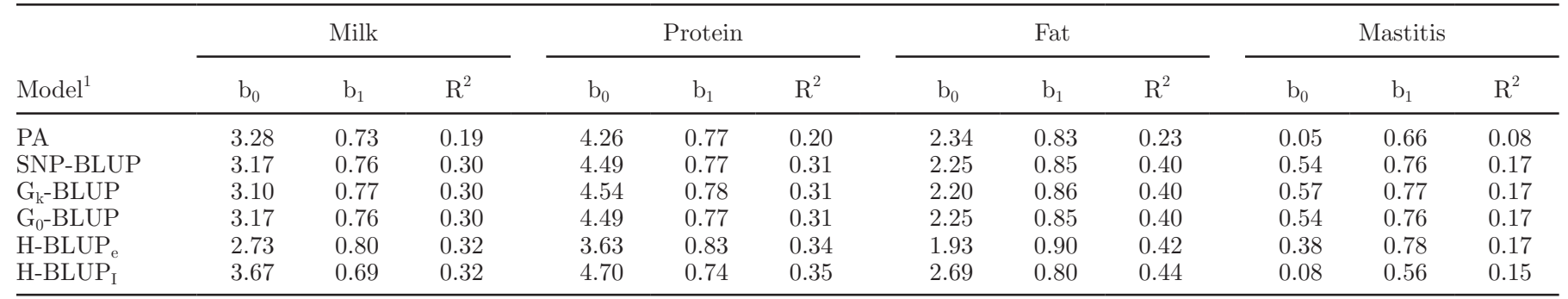

${ }^{1} \mathrm{SNP}-\mathrm{BLUP}=\mathrm{SNP}$ marker BLUP model; $\mathrm{G}_{\mathrm{k}}$-BLUP $=$ BLUP using a scaled genomic relationship matrix; $\mathrm{G}_{0}-\mathrm{BLUP}=\mathrm{BLUP}$ using an unscaled genomic relationship matrix; and $\mathrm{H}-\mathrm{BLUP}=$ one-step approach. 
centage units for milk, and protein, 9 percentage units for mastitis, and 17 percentage units for fat production.

For production traits, validation reliabilities of SNPBLUP and the G-BLUP models were the same, but H-BLUP had on average 2 percentage units higher reliability. The H-BLUP models with estimated variance components or variance components from national evaluations performed equally. Slight difference in $b_{1}$ values between the $2 \mathrm{H}$-BLUP models hinted that the use of the variance components with lower lambda values could overrate the value of genomic information. The validation reliabilities for mastitis were lower than for the other traits, being 0.17 for all genomic prediction models. One reason is that the DRP values were more uncertain among the reference and candidate bulls because average DRP reliability was lower for mastitis than for the production traits. Thus, both the prediction using the reference bull information and the calculated validation reliabilities using the candidate bulls were based on less accurate information. For mastitis, H-BLUP did not perform any better than the other genomic models. However, for mastitis, the validation reliability of PA was very low, and so the relative improvement in the validation reliability by all genomic models was higher than for the production traits.

Only a few papers describe the validation reliability of GEBV based on field data. Su et al. (2012) reported, in RDC, reliabilities for 15 traits and found that reliability of DGV was on average 11 percentage units higher than reliability of PI. Compared with our results, Su et al. (2012) reported on average 5.3 percentage units higher validation reliabilities for G-BLUP and 5.4 percentage units higher reliabilities for the onestep approach. However, when considering differences of genomic model estimates to PA (or PI; Su et al., 2012), these numbers are 2.7 and 2.9 percentage units. This illustrates difficulty of comparing results between studies even within the same breed. We used 2 data sets in our validation and $\mathrm{Su}$ et al. (2012) used one set of EBV, which were then deregressed twice to get reference and validation data sets. Moreover, they used $w=0.20$ in H-BLUP, which improved validation results and especially $b_{1}$ in H-BLUP.

Generally, validation reliabilities seem to be lower for RDC than for Holstein. For Nordic Holstein, validation reliabilities of GEBV in production and mastitis traits ranged from 0.41 to 0.49 , which were 3 to 19 percentage units greater than reliabilities of PA (Su et al., 2010). Hayes et al. (2009a) reported that reliabilities of GEBV ranged from 0.18 to 0.53 for Australian Holstein population, which were 2 to 18 percentage units higher than reliabilities by PA. VanRaden et al. (2009) investigated reliability of GEBV in North American Holstein and reported that for production traits reliabilities of the index combining GEBV and PA was on average $31 \%$ higher than reliability by PA. One reason for the lower validation results in RDC is probably the heterogeneity of the RDC population. The Danish RDC population has a heterogeneous breed history and can be genetically slightly different from the Finnish and Swedish populations. The accuracy of genomic predictions also depends on the amount of linkage disequilibrium (LD) between QTL. The amount of LD is a function of effective population size, with small population size creating large amounts of LD (Hayes et al., 2003; Goddard, 2009). Accordingly, it has been estimated that the effective population size is larger in RDC breeds than in Holstein, and consequently the LD is low compared with that in Holstein (Rius-Vilarrasa et al., 2011).

In the present study, the validation reliabilities of the genomic prediction models were on average $45 \%$ higher than the reliability of PA. Somewhat lower validation reliability of mastitis compared with those of production traits could be due to the lower heritability of mastitis. It has been reported that lower heritability traits, especially with fewer daughters per bull, have lower reliabilities (Luan et al., 2009; Guo et al., 2010).

The H-BLUP used in this study is easy to implement and it improves evaluations of genotyped animals. The H-BLUP model has several advantages. In H-BLUP, the information from nongenotyped animals is included in genomic predictions of their genotyped relatives; thus, H-BLUP will lead directly into GEBV. However, some results have indicated that incorrect weighting of polygenic and genomic components may bias the genomic predictions from H-BLUP (Forni et al., 2011). Validation of H-BLUP indicated that the one-step method does not increase accuracy of evaluation of nongenotyped individuals as much as expected. When examining the validation reliabilities of the 200 youngest candidate bulls with or without marker information, we found that when the marker information was not available for the bulls themselves, the validation reliability was, on average, only $2 \%$ higher with H-BLUP than with PA for the production traits (Table 5 ), regardless of using estimated variance components or variance components from national evaluation. For mastitis, however, the reliability of genetic evaluation for the 200 candidates using H-BLUP was less than that of traditional PA. Still, the results for production traits indicate a slight advantage of the one-step method over a traditional BLUP model even for nongenotyped candidates. This may be because most sires of the youngest candidate bulls had also been genotyped. Thus, young candidates had genomic information also through their sires.

The one-step H-BLUP used in the present study is not a real single-step approach (Legarra et al., 2009; 
Table 5. Intercept $\left(\mathrm{b}_{0}\right)$, regression coefficient $\left(\mathrm{b}_{1}\right)$, validation reliability of parent averages $(\mathrm{PA})$, and one-step H-BLUP with (One-Step_all) or without (One-Step_cut) genomic information for the 200 youngest candidate bulls by trait

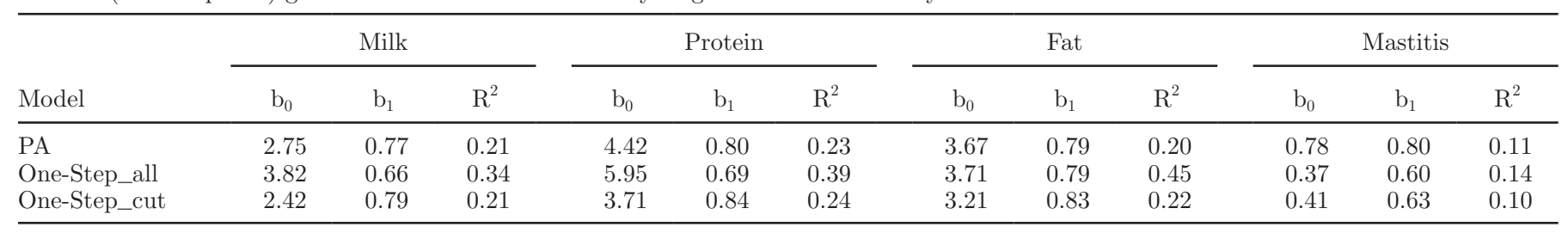

Misztal et al., 2009; Aguilar et al., 2010) as the GEBV were not based on original phenotypic records of the whole population but on DRP. By using original phenotypic data, uncertainty arising during the deregression could be avoided. The real single-step approach has a higher computational demand because the original phenotypic data set in dairy cattle is usually very large, and complicated statistical models are required to account for various effects (Su et al., 2012). However, because the national EBV are already computed using these models, substitution of the numerator relationship matrix $\mathbf{A}$ by the genomic-enhanced $\mathbf{H}$ matrix would allow an easy way to improve current genetic evaluations, as implicated. Additional computational costs in the one-step approach may be lower than in a 2-step G-BLUP approach.

\section{CONCLUSIONS}

We compared genomic predictions and validation reliabilities of SNP-BLUP, G-BLUP, and H-BLUP in a Nordic RDC population. The different genomic methods gave comparable results. For candidate bulls, the SNP-BLUP and G-BLUP models gave the same DGV but we observed a difference in GEBV from H-BLUP. In general, reliability of genomic predictions was $45 \%$ higher than reliability of traditional PA, averaged over the production and mastitis traits. For all traits, SNP-BLUP and G-BLUP gave the same validation reliabilities but H-BLUP had slightly higher validation reliabilities than the other models. One reason for differences in results from H-BLUP is the $10 \%$ polygenic weighting when using blended $\mathbf{G}$ to create the $\mathbf{H}$ matrix. Although the comparisons are only indicative, the H-BLUP method seems to give more accurate results than G-BLUP or SNP-BLUP and has several advantages. With H-BLUP, all animals are considered jointly, and by combining pedigree and genomic information, GEBV are computed directly.

\section{ACKNOWLEDGMENTS}

This work was a part of the Genomic Selection project originally established by Aarhus University and the
Nordic cattle breeding organizations Viking Genetics (Randers, Denmark), Nordic Cattle Genetic Evaluation (Aarhus, Denmark), and Faba (Hollola, Finland). They are acknowledged for providing the genotype data and the EBV data. Project costs were funded by the Finnish Ministry of Agriculture and Forestry (project Genomic information in genetic evaluations and breeding programs by MAKERA) and by Faba (TEKES project: Use of genomic information in cattle breeding).

\section{REFERENCES}

Aguilar, I., I. Misztal, D. L. Johnson, A. Legarra, and S. Tsuruta. 2010. Hot topic: A unified approach to utilize phenotypic, full pedigree, and genomic information for genetic evaluation of Holstein final score. J. Dairy Sci. 93:743-752.

Aguilar, I., I. Misztal, A. Legarra, and S. Tsuruta. 2011. Efficient computation of the genomic relationship matrix and other matrices used in single-step evaluation. J. Anim. Breed. Genet. 128:422428.

Anderson, E., Z. Bai, C. Bischof, J. Demmel, J. Dongarra, J. du Croz, A. Greenbaum, S. Hammarling, J. D. McKenney, and D. Sorensen. 1990. LAPACK: A portable linear algebra library for high-performance computers. Computer Science Dept. Technical Report CS90-105. University of Tennessee, Knoxville.

Christensen, O. F., and M. S. Lund. 2010. Genomic prediction when some animals are not genotyped. Genet. Sel. Evol. 42:2.

Cole, J. B., P. M. VanRaden, J. R. O'Connell, C. P. Van Tassell, and T. S. Sonstegard. 2009. Distribution and location of genetic effects for dairy traits. J. Dairy Sci. 92:2931-2946.

Fikse, W. F., and G. Banos. 2001. Weighting factors of sire daughter information in international genetic evaluations. J. Dairy Sci. 84:1759-1767.

Forni, S., I. Aguilar, and I. Misztal. 2011. Different genomic relationship matrices for single-step analysis using phenotypic, pedigree and genomic information. Genet. Sel. Evol. 43:1.

Garrick, D. J., J. F. Taylor, and R. L. Fernando. 2009. Deregressing estimated breeding values and weighting information for genomic regression analyses. Genet. Sel. Evol. 41:55.

Gianola, D., R. L. Fernando, and A. Stella. 2006. Genomic-assisted prediction of genetic value with semiparametric procedures. Genetics $173: 1761-1776$.

Gianola, D., and B. C. H. M. van Kamm. 2008. Reproducing kernel Hilbert spaces regression methods for genomic prediction of quantitative traits. Genetics 178:2289-2303.

Goddard, M. 1985. A method of comparing sires evaluated in different countries. Livest. Prod. Sci. 13:321-331.

Goddard, M. 2009. Genomic selection: Prediction of accuracy and maximisation of long term response. Genetica 136:245-257.

Goddard, M. E., and B. J. Hayes. 2009. Mapping genes for complex traits in domestic animals and their use in breeding programmes. Nat. Rev. Genet. 10:381-391.

Grisart, B., F. Farnir, L. Karim, N. Cambisano, and J. J. Kim. 2004. Genetic and functional confirmation of the causality of the DGAT1 
K232A quantitative trait nucleotide in affecting milk yield and composition. Proc. Natl. Acad. Sci. USA 101:2398-2403.

Guillaume, F., S. Fritz, D. Boichard, and T. Druet. 2008. Short communication: Correlations of marker-assisted breeding values with progeny-test breeding values for eight hundred ninety-nine French Holstein bulls. J. Dairy Sci. 91:2520-2522.

Guo, G., M. S. Lund, Y. Zhang, and G. Su. 2010. Comparison between genomic predictions using daughter yield deviation and conventional estimated breeding value as response variables. J. Anim. Breed. Genet. 127:423-432.

Harris, B. L., and D. L. Johnson. 2010. Genomic predictions for New Zealand dairy bulls and integration with national genetic evaluation. J. Dairy Sci. 93:1243-1252.

Hayes, B. J., P. J. Bowman, A. J. Chamberlain, and M. E. Goddard. 2009a. Invited review: Genomic selection in dairy cattle: Progress and challenges. J. Dairy Sci. 92:433-443.

Hayes, B. J., P. M. Visscher, and M. E. Goddard. 2009b. Increased accuracy of artificial selection by using the realized relationship matrix. Genet. Res. (Camb.) 91:47-60.

Hayes, B. J., P. M. Visscher, H. C. McPartlan, and M. E. Goddard. 2003. Novel multilocus measure of linkage disequilibrium to estimate past effective population size. Genome Res. 13:635-643.

Illumina Inc. 2005. Illumina GenCall Data Analysis Software-GenCall software algorithms for clustering, calling, and scoring genotypes. Illumina. Pub. No. 370-2004-009. Illumina Inc., San Diego, CA.

Jairath, L., J. C. M. Dekkers, L. R. Schaeffer, Z. Liu, and E. B. Burnside. 1998. Genetic evaluation for herd life in Canada. J. Dairy Sci. 81:550-562.

Legarra, A., I. Aguilar, and I. Misztal. 2009. A relationship matrix including full pedigree and genomic information. J. Dairy Sci. 92:4656-4663.

Luan, T., J. A. Woolliams, L. Sigbjorn, M. Kent, M. Svendsen, and T. H. E. Meuwissen. 2009. The accuracy of genomic selection in Norwegian Red cattle assessed by cross-validation. Genetics 183:1119-1126.

Mäntysaari, E., Z. Liu, and P. VanRaden. 2010. Interbull validation test for genomic evaluations. Interbull Bull. 40:1-5.

Meuwissen, T. H. E., and M. E. Goddard. 2004. Mapping multiple QTL using linkage disequilibrium and linkage analysis information and multitrait data. Genet. Sel. Evol. 36:261-279.

Meuwissen, T. H. E., B. J. Hayes, and M. E. Goddard. 2001. Prediction of total genetic value using genome-wide dense marker maps. Genetics 157:1819-1829.

Misztal, I., A. Legarra, and I. Aguilar. 2009. Computing procedures for genetic evaluation including phenotypic, full pedigree, and genomic information. J. Dairy Sci. 92:4648-4655.

Misztal, I., S. Tsuruta, T. Strabel, B. Auvray, T. Druet, and D. H. Lee. 2002. Blupf90 and related programs (BGF90). Proc. 7th World Congr. Genet. Appl. Livest. Prod., Montpellier, France. 33:743744 .
Ostersen, T., O. F. Christensen, M. Henryon, B. Nielsen, G. Su, and P. Madsen. 2011. Deregressed EBV as the response variable yield more reliable genomic predictions than traditional EBV in purebred pigs. Genet. Sel. Evol. 43:38.

Rius-Vilarrasa, E., T. Iso-Touru, I. Strandén, N. Schulman, B. Guldbrandtsen, E. Strandberg, M. S. Lund, J. Vilkki, and W. F. Fikse. 2011. Characterization of linkage disequilibrium in a Danish, Swedish and Finnish Red Breed cattle population. Page 177 in Proc. 62nd Annu. Mtg. Eur. Fed. Anim. Sci., Stavanger, Norway. Wageningen Academic Publishers, Wageningen, the Netherlands.

Schaeffer, L. R. 2001. Multiple trait international bull comparisons. Livest. Prod. Sci. 69:145-153.

Scheet, P., and M. Stephens. 2006. A fast and flexible statistical model for large-scale population genotype data: Applications to inferring missing genotypes and haplotypic phase. Am. J. Hum. Genet. $78: 629-644$.

Strandén, I., and O. F. Christensen. 2011. Allele coding in genomic evaluation. Genet. Sel. Evol. 43:25.

Strandén, I., and D. Garrick. 2009. Technical note: Derivation of equivalent computing algorithms for genomic predictions and reliabilities of animal merit. J. Dairy Sci. 92:2971-2975.

Strandén, I., and M. Lidauer. 1999. Solving large mixed models using preconditioned conjugate gradient iteration. J. Dairy Sci. 82:2779-2787.

Strandén, I., and E. A. Mäntysaari. 2010. A recipe for multiple trait deregression. Interbull Bull. 42:21-24.

Su, G., B. Guldbrandtsen, V. R. Gregersen, and M. S. Lund. 2010 Preliminary investigation on reliability of genomic estimated breeding values in the Danish Holstein population. J. Dairy Sci. 93:1175-1183.

Su, G., P. Madsen, U. S. Nielsen, E. A. Mäntysaari, G. P. Aamand, O. F. Christensen, and M. S. Lund. 2012. Genomic prediction for the Nordic Red Cattle using one-step and selection index blending approaches. J. Dairy Sci. 95:909-917.

VanRaden, P. M. 2008. Efficient methods to compute genomic predictions. J. Dairy Sci. 91:4414-4423.

VanRaden, P. M., and P. G. Sullivan. 2010. International genomic evaluation methods for dairy cattle. Genet. Sel. Evol. 42:7.

VanRaden, P. M., C. P. Van Tassell, G. R. Wiggans, T. S. Sonstegard, and R. D. Schnabel. 2009. Invited review: Reliability of genomic predictions for North American Holstein bulls. J. Dairy Sci. 92:16-24.

Villanueva, B., R. Pong-Wong, J. Fernández, and M. A. Toro. 2005. Benefits from marker-assisted selection under an additive polygenic genetic model. J. Anim. Sci. 83:1747-1752.

Yang, J., S. H. Lee, M. E. Goddard, and P. M. Visscher. 2011. Gcta: A tool for genome-wide complex trait analysis. Am. J. Hum. Genet. 88:76-82. 